\title{
A new conservative finite-difference scheme for anisotropic elliptic problems in bounded domain
}

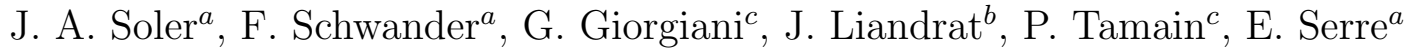 \\ ${ }^{a}$ Aix Marseille Univ., CNRS, Centrale Marseille, M2P2, Marseille, France. \\ ${ }^{b}$ Aix Marseille Univ., CNRS, Centrale Marseille, I2M, Marseille, France \\ ${ }^{c}$ IRFM, CEA Cadarache, F-13108 St. Paul-lez-Durance, France
}

\begin{abstract}
Highly anisotropic elliptic problems occur in many physical models that need be solved numerically, and in this case regular, structured mesh methods are generally not designed to provide an optimal discretization. Starting from the experience gained in the simulation of turbulence in magnetized fusion plasmas, we propose a new finite-difference scheme based on aligned interpolations that can be applied for the resolution of a large class of highly anistropic elliptic problems beyond magnetic fusion. Numerical tests on manufactured solutions show accurate and stable numerical approximations in not aligned cartesian or cylindrical grids. For a given accuracy, results show that mesh points can be saved with respect to non-aligned approaches when the anisotropy is increased. Compared with the existing literature, this new scheme is shown to be conservative, and is able to deal accurately and efficiently with any Dirichlet, Neuman or Robin boundary conditions in the direction of anisotropy at the border of the domain.
\end{abstract}

Keywords: Anisotropic operators - Conservative finite-difference scheme - Aligned interpolation 


\title{
A new conservative finite-difference scheme for anisotropic elliptic problems in bounded domain
}

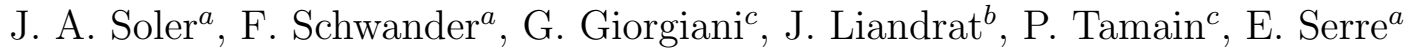 \\ a Aix Marseille Univ., CNRS, Centrale Marseille, M2P2, Marseille, France. \\ ${ }^{b}$ Aix Marseille Univ., CNRS, Centrale Marseille, I2M, Marseille, France \\ ${ }^{c}$ IRFM, CEA Cadarache, F-13108 St. Paul-lez-Durance, France
}

\section{Introduction}

Elliptic partial differential systems are ubiquitous in physical models and numerical simulations. They occur in fluid models used in mechanics, geophysics, plasma physics, but also in other fields of research as in microelectronics, optics, image processing, and so on, the list being not exhaustive.

A typical problem to solve with appropriate boundary conditions is the Poisson's equation that writes as:

$$
\boldsymbol{\nabla} \cdot([K] \cdot \nabla) T=S, \quad \text { in } \Omega \subset \mathbb{R}^{3}
$$

where $[K]$ is the diffusion tensor that can be written in a coordinate system whose axes coincide with the principal axes of the tensor such as:

$$
\left[\begin{array}{ccc}
K_{b_{\|}} & 0 & 0 \\
0 & K_{b_{\perp}} & 0 \\
0 & 0 & K_{z}
\end{array}\right]
$$

where $K_{b_{\|}}, K_{b_{\perp}}$ and $K_{z}$ are given functions along these axes.

In many configurations the isotropy of the problem can be broken, and a preferred direction (direction of anisotropy) is thus introduced that leads to an anisotropic diffusion tensor. Regular, structured mesh methods for conservation laws are generally not designed to discretize such anisotropic operators in an optimal way. They feature inherently preferred directions which can introduce systematic errors, even at high resolution, if physical anisotropies are not aligned with the grid axes. The discretization can in particular produce a significant spurious numerical diffusion in the direction orthogonal to the anisotropy direction, which can significantly impact the perpendicular dynamics [? ]. As recalled in van Es et al. [1], other problems can also arise as the non-positivity near 
high gradients [? ] or the convergence stagnation or loss of the solution $[2,3]$. Such spurious effects can be reduced by using high-order approximations for contribution related to the direction of anisotropy but larger stencils are then required.

Many mathematical and numerical works have been devoted to the discretization of anisotropic diffusion operators (see a quite exhaustive list of references in van Es et al. [1]). More specifically in the frame of finite-difference methods, support operator methods (SOM) [4, 5], also known as Mimetic finite-difference [6], allow to conserve the self-adjointness of the divergence and the flux operator and the positive definiteness of the continuum problem when discretizing second-order partial differential equations. Hyman et al. [6], set the conditions to define Dirichlet, Neuman and Robin boundary conditions preserving SOM properties. In Morel et al. [7] a new local version of SOM was proposed, which yields a sparse matrix for diffusion equation.

The initial motivation of this work being related to magnetic confinement fusion and to the simulation of edge plasma turbulence, the direction of anisotropy corresponds to the parallel direction along the magnetic fied lines that defines the privileged flow direction (the magnitudes of the two components of the magnetic field in the toroidal and poloidal directions verifiy $\left|B_{p}\right| \ll\left|B_{t}\right|$ in a tokamak) along which the governing equations are usually projected [8]. For the simulation of magnetized fusion plasmas, Gunter et al. [3] proposed a finite-difference method with SOM conditions in not-aligned coordinates in rectangular grids, reducing the perpendicular pollution due to the parallel discretization. Ottaviani [9] and Hariri \& Ottaviani [10] introduced the Flux-Coordinate Independent approach, a field-aligned approach in not aligned cartesian and polar grids, interpolating the field value on the magnetic field line (parallel diffusion direction). This method is able to reduce the number of grid points in rectangular and cylindrical grids (reducing drastically the grid unknowns). The parallel numerical diffusion is shown to decrease, and to be subdominant with respect to the perpendicular second-order dynamics calculation based on a classical Arakawa scheme [11]. In Stegmeir et al. [12] the Field Line Map (FLM) approach is presented. Based on the magnetic field lines trace geometry, Stegmeir et al. present an integration method for the gradient operator from interpolated field values in the magnetic field lines, before deriving the full diffusion operator with SOM. The resulting approach presents lower numerical diffusion, higher convergence tendency and the grid unknowns reduction presented in [9]. Finally van Es et al. [1] compare the accuracy properties of Gunter et al.'s schemes with field lines tracking approaches in regular cartesian grids using the surrounding points. The comparison is made in anisotropic cases. What's the conclusion?????????????-i In general, aligned approaches present a natural operator discretization generaly not aligned with a grid for highly anisotropic problems in a nu parler des résultats, range d'applications (grille, anisotropie, etc),

In this work, we propose a new finite-difference scheme based on interpolations aligned with the direction of anisotropy. Compared with the existing literature, it is shown to be conservative and able to deal accurately with non-periodic boundary conditions 
in the anisotropy direction at the border of the domain. The paper is organised as follows: in Section 2 the mathematical model related to an elliptic problem is presented, the numerical scheme is detailed in Section 3 for the discretization of the parallel and perpendicular operators in the interior of the computational domain and in Section 5 for the boundaries. In Section 6, the problem of grid limitation on not aligned grids is addressed depending on the numerical approach is aligned or not. Finally, results showing the accuracy and the efficiency of the new scheme are presented in Section 7 on the elliptic problem Eq. 3 and in Section 8 for the special case of Poisson's equation $(\mu=0)$ depending on Dirichlet, Neuman or Robin boundary conditions are used.

\section{Mathematical model}

An elliptic problem with general boundary conditions is considered as :

$$
\left\{\begin{array}{cc}
-\boldsymbol{\nabla} \cdot[K] \nabla T+\mu T=S & \text { on } \Omega \\
\beta \nabla_{\|} T+\gamma T=s & \text { in } \Gamma
\end{array}\right.
$$

where $\Omega$ is a domain of boundary $\partial \Omega$ in $\mathbb{R}^{3}$ provided a cartesian frame of reference $(x, y, z)$. We assume the variables of the problem satisfy the usual ellipticity and regularity assumptions. $\mu$ is a positive (or zero) constant, and $S$ and $s$ are some given source terms. $\beta$ and $\gamma$ can be equal to zero or one, depending on Dirichlet, Neumann or Robin boundary conditions are considered. This problem allows us to decollerate the treatment of the equation in the interior of the domain (periodic domain) and at the boundaries.

The anisotropy of the problem is taken into account via the definition of the diffusion tensor $[K]$. In the present work the anisotropy direction is fixed, and is aligned with the first main axis of the tensor denoted $K_{b_{\|}}$.

Thus, in the $(x, y, z)$ non aligned-coordinate the system can be written as:

$$
\left.-\boldsymbol{\nabla} \cdot[\pi]\left[\begin{array}{ccc}
\frac{1}{\epsilon} K_{b_{\|}} & 0 & 0 \\
0 & K_{b_{\perp}} & 0 \\
0 & 0 & K_{z}
\end{array}\right][\pi]^{-1}\right]\left[\begin{array}{l}
\partial \mathbf{T} / \partial x \\
\partial \mathbf{T} / \partial y \\
\partial \mathbf{T} / \partial z
\end{array}\right]+\mu[\mathbb{I}][\mathbf{T}]=S
$$

where $[\mathbb{I}]$ is the identity matrix and $[\pi]$ defines the rotation matrix of angle $\alpha$ :

$$
[\pi]=\left[\begin{array}{ccc}
\cos \alpha & -\sin \alpha & 0 \\
\sin \alpha & \cos \alpha & 0 \\
0 & 0 & 1
\end{array}\right]
$$

In this work, we will assume that $K_{b_{\|}}(x, y), K_{b_{\perp}}(x, y)$ and $K_{z}(z)$ are terms with comparable order of magnitude, and $\epsilon$ is a small parameter defining the anisotropy. $\alpha$ is the angle that the unit vector $\mathbf{b}$ along the privileged direction (Fig. 1) subtends with the $x$-axis. Thus, in the $(x, y)$-plane, $\mathbf{b}=(\cos \alpha, \sin \alpha)^{t}$. Gradients along the parallel and perpendicular direction are then defined as $\nabla_{\|}=\mathbf{b} \cdot \boldsymbol{\nabla}$ and $\nabla_{\perp}=\boldsymbol{\nabla}-\boldsymbol{b} \nabla_{\|}$, respectively. 
Great simplifications can be obtained by choosing a coordinate system whose axes coincide with the principal axes of $[K]$. If we consider the $\left(b_{\|}, b_{\perp}, b_{z}\right)$ aligned-coordinate system, the algebraic system Eq.4 simplifies as:

$$
-\boldsymbol{\nabla} \cdot\left[\begin{array}{ccc}
\frac{1}{\epsilon} K_{b_{\|}} & 0 & 0 \\
0 & K_{b_{\perp}} & 0 \\
0 & 0 & K_{z}
\end{array}\right]\left[\begin{array}{c}
\partial \mathbf{T} / \partial b_{\|} \\
\partial \mathbf{T} / \partial b_{\perp} \\
\partial \mathbf{T} / \partial z
\end{array}\right]+\mu[\mathbb{I}][\mathbf{T}]=S
$$

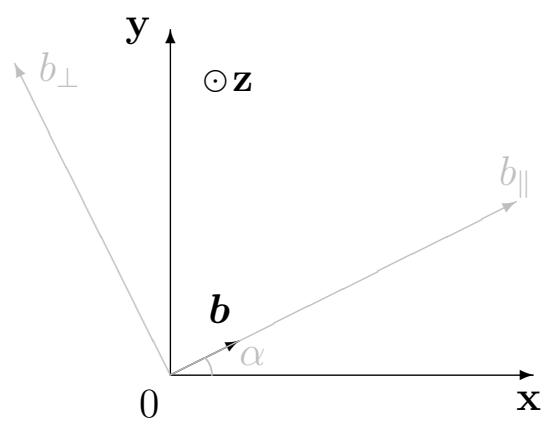

Figure 1: Directions of the principal axes of the diffusion tensor in the $(x, y)$-plane. $\alpha$ defines the misalignment angle of the principal axes with respect to grid points directions.

\section{Numerical discretization in the interior of the domain}

The computational domain is the cube $] 0,2 \pi[\times] 0,2 \pi[\times] 0,2 \pi[$ in the $(x, y, z)$ directions, respectively. It is considered as open, the discretization of the boundary conditions at the border of the domain being considered thereafter.

\subsection{Grid definition}

The grid is structured and uniform. Each cell in the grid can be addressed by indexes $(i, j, k)$, and each vertex has coordinates $x_{i}=i\left(2 \pi / N_{x}\right), y_{j}=j\left(2 \pi / N_{y}\right), z_{k}=k\left(2 \pi / N_{z}\right)$ for $(i, j, k) \in] 1, N_{x}[\times] 1, N_{y}[\times] 1, N_{z}\left[\right.$, where $N_{x}, N_{y}, N_{z}$ is the number of points in each direction. Distances between grid points are defined as $\Delta x=x_{i+1}-x_{i}, \Delta y=y_{j+1}-y_{j}$ and $\Delta z=z_{k+1}-x_{k}$.

In the following, the discretization will be oriented, the local sense of $\boldsymbol{b}$ defining the positive sense at any $(i, j, k)$ point. Quantities to discretize may thus eventually be superscripted with + or - when needed. 


\subsection{A conservative discretization of the parallel gradient $\nabla_{\|}$}

The discretization is made conservative by using a finite-volume formulation. By simplicity, we will assume $\nabla \cdot \boldsymbol{b}=0$. If $\boldsymbol{b}$ was not divergence free, it would be always possible to find a divergence-free vector field everywhere co-linear to $\boldsymbol{b}$. Under this assumption, the following integral definition of $\nabla_{\|}$can be used on each control volume $K$, of volume $V$ and surface $S$ that allows us to estimate the parallel gradient from the flux through $S$ :

$$
\nabla_{\|} T=\nabla \cdot(T \boldsymbol{b}) \equiv \lim _{V(K) \rightarrow 0} \frac{1}{V(K)} \int_{S} T \boldsymbol{b} \cdot \boldsymbol{n} d S
$$

The control volume $K$ around each grid point $T_{i, j, k}$ is defined by the polygon limited in $\left(i, j \pm \frac{1}{2}, k \pm \frac{1}{2}\right)$ in the $y-z$-plane $\mathcal{X}_{i}$, and extruded along the parallel direction up to the planes $\mathcal{X}_{i \pm \frac{1}{2}}$ (Fig. 2). At these planes, it contacts neighbouring control volumes defined from grid points located in both adjacent planes $\mathcal{X}_{i+1}$ and $\mathcal{X}_{i-1}$. In the following, we will only consider by simplicity neighbouring control volumes defined in $\mathcal{X}_{i+1}$, the discretization being similar for control volumes defined in $\mathcal{X}_{i-1}$.

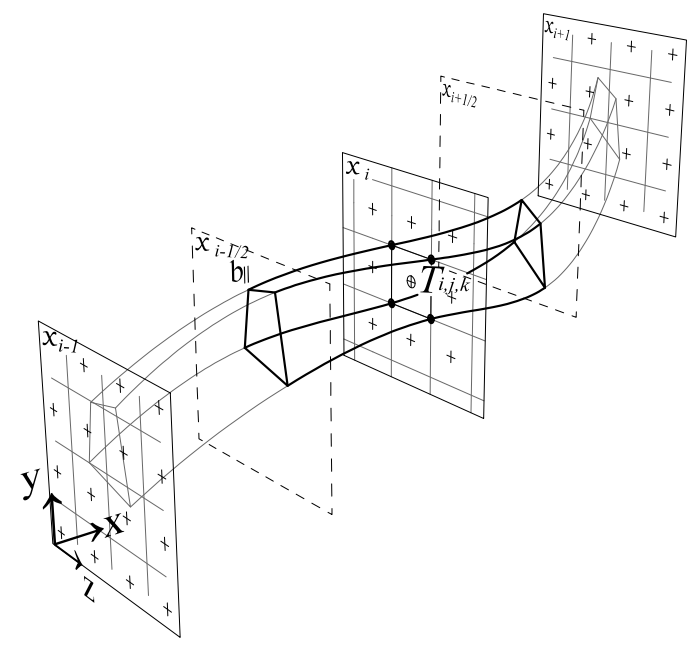

Figure 2: Sketches of control volumes used in this work. In black full line the control volumes defined around $T_{i, j, k} \in \mathcal{X}_{i}$. In grey, the neighboring control volumes in $\mathcal{X}_{i \pm \frac{1}{2}}$. General case with $\boldsymbol{b}(x, y, z)$.

The contact surfaces between the control volume and its $N$ neighbors are denoted $a_{p}, p=1, \ldots, N$ (Fig. 3). For each contact surface $a_{p}$, we consider the line passing by its geometric center that follows the parallel direction, as illustrated in Fig. 3b. It intercepts the two planes $\mathcal{X}_{i}$ and $\mathcal{X}_{i+1}$ at two points of coordinates $\left(x_{i}, y^{-}, z^{-}\right)$and $\left(x_{i+1}, y^{+}, z^{+}\right)$, where $\left(y^{ \pm}, z^{ \pm}\right)$can be defined between $\left(x_{i+1 / 2}\right)$ and $\left(x_{i \pm 1}\right)$ as : 


$$
y^{ \pm}=y+\int_{x_{i+1 / 2}}^{x_{i \pm 1}} \frac{b_{y}}{b_{x}} d x, \quad z^{ \pm}=z+\int_{x_{i+1 / 2}}^{x_{i \pm 1}} \frac{b_{z}}{b_{x}} d x
$$

$b_{x}, b_{y}$ and $b_{z}$ being the components of $\boldsymbol{b}$ in the Cartesian frame $(x, y, z)$.

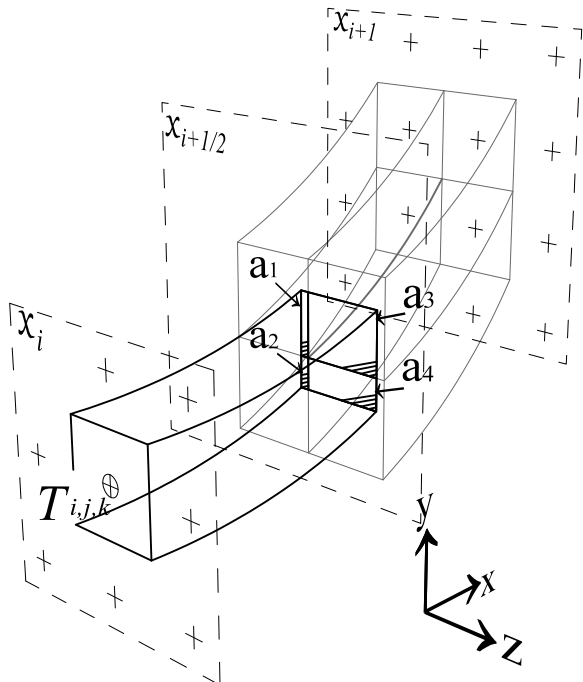

(a)

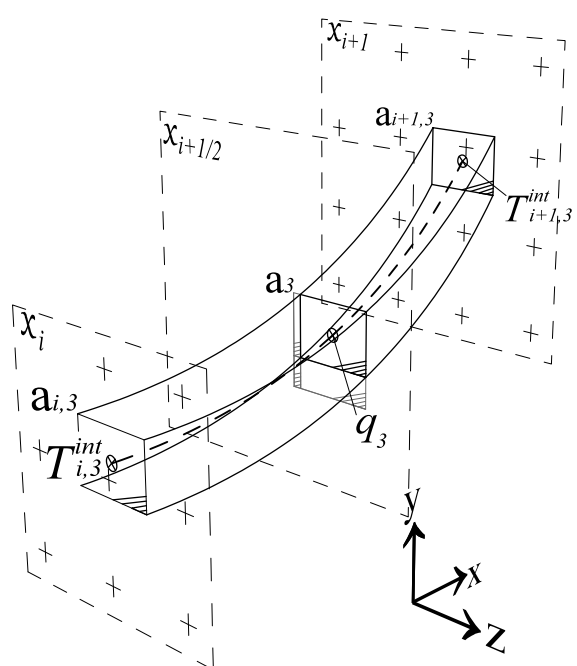

(b)

Figure 3: Zoom on Fig. 2 showing contact surfaces between control volumes for $N=4(\boldsymbol{b}(x)$ here for simplicity) (a), and construction of a control volume used to evaluate the parallel flux $q_{3}$ at $x_{i+1 / 2}$ through the specific surface $a_{3}(\mathrm{~b})$.

The field value at these points are obtained by interpolation of the field values at the surrounding points in the corresponding planes. We can write for each $p$ :

$$
\begin{aligned}
T_{i, p}^{i n t} & =f_{i, p}^{i n t}\left(\left\{T_{i, j, k}\right\}_{i, j=1, \ldots N_{y}, k=1, \ldots N_{z}}\right) \\
T_{i+1, p}^{i n t} & =f_{i+1, p}^{\text {int }}\left(\left\{T_{i+1, j, k}\right\}_{i, j=1, \ldots N_{y}, k=1, \ldots N_{z}}\right)
\end{aligned}
$$

Where $f_{i, p}^{i n t}$ and $f_{i+1, p}^{\text {int }}$ represents the linear interpolation function applied in the grid points. For a lineal interpolation order in $y_{j}<y<y_{j+1}$ and $z_{k}<z<z_{k+1}$ points leads:

Thus, the second-order local flux balance through the plane $a_{p}$ can be discretized as:

$$
\left(\nabla_{\|} T\right)_{p}=\frac{1}{\widehat{\Delta V_{p}}}\left(T_{i+1, p}^{i n t} a_{i+1, p} b_{i, p} \cdot n_{i, p}-T_{i, p}^{i n t} a_{i, p} b_{i+1, p} \cdot n_{i+1, p}\right)
$$

Where $\widetilde{\Delta V_{p}}$ is the volume obtained by integrating the surface $a_{p}$ along the parallel direction between $\mathcal{X}_{i}$ and $\mathcal{X}_{i+1}$. 
The previous discretization can be rewritten as a matrix product of a numerical application $q_{p, \lambda}$ to the discretized field for the whole domain as follows:

$$
\left(\nabla_{\|} T\right)_{p}=\sum_{\lambda} q_{p, \lambda} T_{\lambda}
$$

Where for clarity $\lambda=(i-1) \cdot N_{y} \cdot N_{z}+(j-1) \cdot N_{z}+k$ labels $(i, j, k)$.

\subsection{Discretization of the parallel Laplacian $\nabla \cdot K_{b_{\|}} \nabla_{\|}$}

The parallel Laplacian is obtained using the support-operator method (SOM) [5]. For any functions $T$ and $\Psi$ as well as suitable boundary conditions (bi-periodic or homogeneous Dirichlet), the Green formula allows us to define the parallel diffusion operator directly from the parallel gradient as:

$$
\left\langle\nabla \cdot[K] \nabla_{\|} T, \Psi\right\rangle=-\left\langle[K] \nabla_{\|} T, \nabla_{\|} \Psi\right\rangle
$$

where $\langle\cdot, \cdot\rangle$ defines the $\mathcal{L}^{2}$-inner product. Though Eq. 13 is unambiguous at the continuous level, it involves two inner products defined in the gradient space (GS) and in the fluxes space (FS) for any functions $f$ and $g$ as:

$$
\begin{aligned}
& \langle f, g\rangle=\sum_{k} f_{k} g_{k} \Delta V_{k} \\
& \widetilde{\langle f, g\rangle}=\sum_{p} f_{p} g_{p} \widetilde{\Delta V_{p}}
\end{aligned}
$$

According to Eq. 11, the inner product in (FS) can be estimated at the discrete level using evaluations of the diffusivity on flux points denoted by $\left\{K_{b_{\|}, p}\right\}$ as:

$$
\left\langle[K] \nabla_{\|} T, \nabla_{\|} \Psi\right\rangle \equiv \sum_{p}\left(K_{b_{\|}, p} \sum_{\lambda} q_{p, \lambda} T_{\lambda}\right) \times\left(\sum_{\mu} q_{p, \mu} \Psi_{\mu}\right) \widetilde{\Delta V_{p}}
$$

For each $\lambda, N$ values of flux are associated and the flux space generated by all $q_{\lambda, p}$ is labeled by $(\lambda, p)$.

As shown in Eq. 11, $q_{\lambda, p}$ in (FS) is estimated from $(G S)$. The adjoint operator defined by the matrix $q_{\lambda, p}$ is a discretization of the divergence operator defined from $(F S)$ into $(G S)$. Then, the operator $\left(\nabla \cdot[K] \nabla_{\|}\right)$is defined from (GS) into (GS).

The left-hand side of Eq. 13 leads at the discrete level to:

$$
\left\langle\nabla \cdot[K] \nabla_{\|} T, \Psi\right\rangle \equiv \sum_{\lambda}\left(\nabla \cdot[K] \nabla_{\|} T\right)_{\lambda} \Psi_{\lambda} \Delta V_{\lambda}
$$

According now to Eqs. 13, 16 and 17, one deduces by identification that: 


$$
\left(\nabla \cdot[K] \nabla_{\|} T\right)_{i}=-\frac{1}{\Delta V_{i}} \sum_{p}\left(K_{b_{\|}, p} q_{p i} \sum_{k} q_{p k} T_{k} \widetilde{\Delta V_{p}}\right)
$$

Upon multiplication by the cell volume, the SOM provides a symmetric discrete matrix since:

$$
A_{\lambda \mu} \Delta V_{\lambda}=\sum_{p} q_{p \lambda} q_{p \mu}[K]_{p} \widetilde{\Delta V_{p}}=\sum_{p} q_{p \mu} q_{p \lambda}[K]_{p} \widetilde{\Delta V_{p}}=A_{\mu \lambda} \Delta V_{\mu}
$$

where $[K]_{p} \widetilde{\Delta V_{p}}=[K \widetilde{\Delta V}]_{p, p}$ is a $p \times p$ diagonal square matrix.

The parallel approach has been described for a full three-dimensional operator. In the following, and according to Eqs. 5 and 2, the problem can be decomposed into a two dimensional problem in the $b_{\|}$and $b_{\perp}$ directions, not aligned with the grid, plus an additional dimension $b_{z}$, aligned with the grid.

The discretization being not aligned with the grid, the points needed for the discretization are shifted with respect to the Cartesian grid. This shift is equal to:

$$
\xi=\left\lfloor\frac{\int_{x_{i}}^{x_{i \pm 1}} \frac{b_{y}}{b_{x}} d x}{\Delta y}\right\rfloor
$$

which simplifies as:

$$
\xi=\left\lfloor\frac{\Delta x}{\Delta y} \tan \alpha\right\rfloor
$$

when the diffusivity tensor is uniform in space.

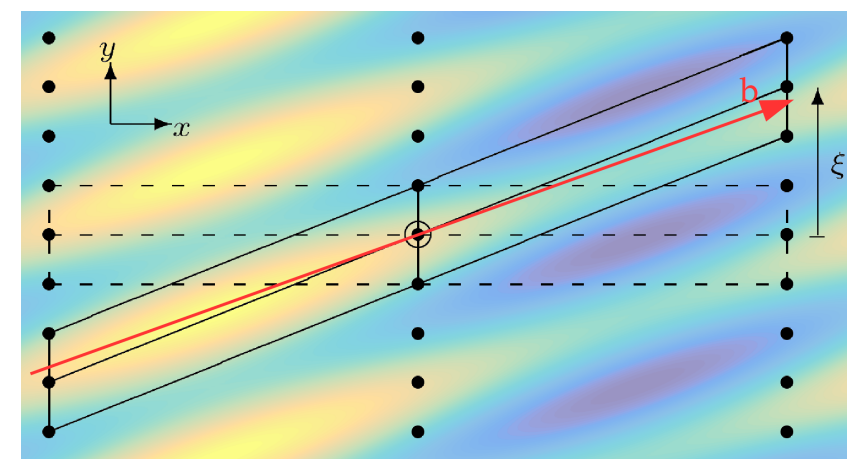

Figure 4: Example of shifted points for a 9-points stencils in the $(x, y)$ plane: not oriented stencil (dashed lines) and oriented stencils (full lines) for a shift $\xi=3$. The arrow along $b$ shows the positive sense. 


\subsection{A conservative discretization of the perpendicular gradient $\nabla_{\perp}$ and Laplacian}

In this paper, we also propose a conservative approach to discretize the perpendicular direction. The perpendicular gradient $\nabla_{\perp}$ at $\left(i, j \pm \frac{1}{2}\right)$ is expressed as a combination of the parallel gradient and of a contribution coming from the gradient in the $y$-direction as follows:

$$
\left(\nabla_{\perp} T_{i, j \pm 1 / 2}\right)=\frac{\nabla_{y} T_{i, j \pm 1 / 2}-\nabla_{\|} T_{i, j \pm 1 / 2} \sin \alpha}{\cos \alpha}
$$

where $\nabla_{\|}$is obtained using an aligned approach as:

$$
\nabla_{\|} T_{i, j \pm 1 / 2}=\frac{T_{i+1, j \pm 1 / 2}^{i n t}-T_{i-1, j \pm 1 / 2}^{i n t}}{d_{\|}^{ \pm}}
$$

where $T_{i \pm 1, j \pm 1 / 2}^{i n t}$ are the values of $T$ obtained by a polynomial interpolation (Vandermonde) in the plane $\mathcal{X}_{i \pm 1}$, and $d_{\|}$is the distance in the parallel direction between two successive planes $\left(\mathcal{X}_{i}-\mathcal{X}_{i \pm 1}\right)$ :

$$
d_{\|}^{ \pm}=\left|\int_{x_{i}}^{x_{i \pm 1}} \frac{\sqrt{b_{x}^{2}+b_{y}^{2}+b_{z}^{2}}}{\left|b_{x}\right|} \mathrm{d} x\right|
$$

The contribution of the gradient in the $y$-direction is easily obtained between two adjacent grid points at $(i, j \pm 1)$ by centered finite-differences:

$$
\begin{aligned}
& \left(\nabla_{y} T_{i, j+1 / 2}\right)=\frac{T_{i, j+1}-T_{i, j}}{\Delta y} \\
& \left(\nabla_{y} T_{i, j-1 / 2}\right)=\frac{T_{i, j}-T_{i, j-1}}{\Delta y}
\end{aligned}
$$

\section{Conservativity}

The conservativity of the new scheme is shown for the parallel operator which is the privileged direction of the problem. The discretization of the perpendicular operator shown previously guarantees the conservativity in this direction. To show the conservativity, a test case avoiding the interpolation step is considered. It is based on a manufactured solution $T_{a}$, constant in the interpolation direction $\left(T_{a}=\sin \left(x_{i}\right)\right)$, and a variable diffusivity such as $1 / \epsilon=K \sin \left(x_{i}\right) \sin \left(y_{j}\right)$. Eq. 3 is solved with $\mu=1$ and with bi-periodic boundary conditions, varying the angle $\alpha=\tan ^{-1}\left(m_{x} / m_{y}\right)$, for $m_{y}=8$ and $m_{x} \in[1,8]$. Only straight parallel diffusion lines are considered. Results are compared to the Flux-Coordinate Independent approach of Ottaviani [9] and Hariri \& Ottaviani [10], referred here as the Ottaviani's scheme. 


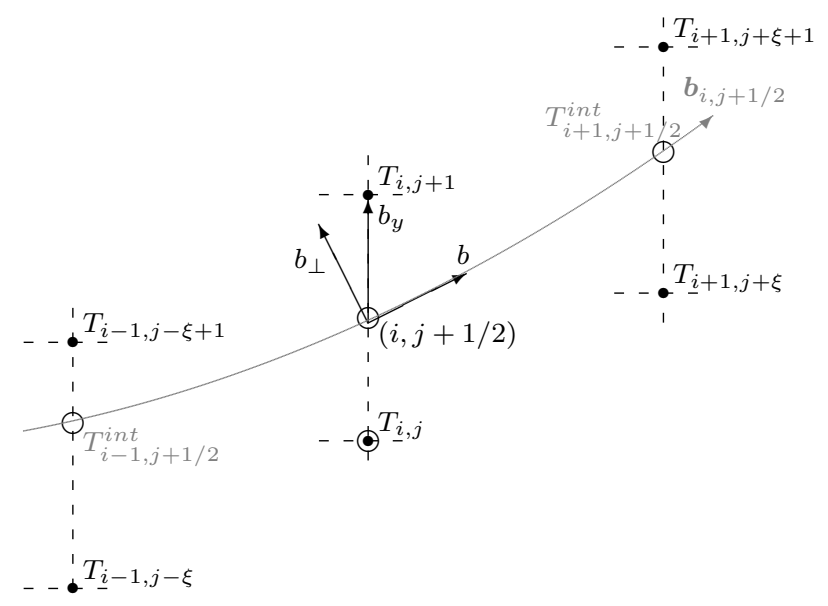

Figure 5: Grid points distribution needed to discretize the perpendicular operator. $T_{i \pm 1, j \pm 1 / 2}^{i n t}$ are the values of $T$ along the parallel direction obtained by a polynomial interpolation (Vandermonde) in the plane $\mathcal{X}_{i \pm 1} . \xi$ is the shift value calculated from Eq. 20.

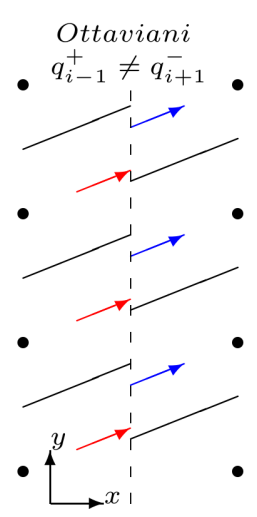

(a)
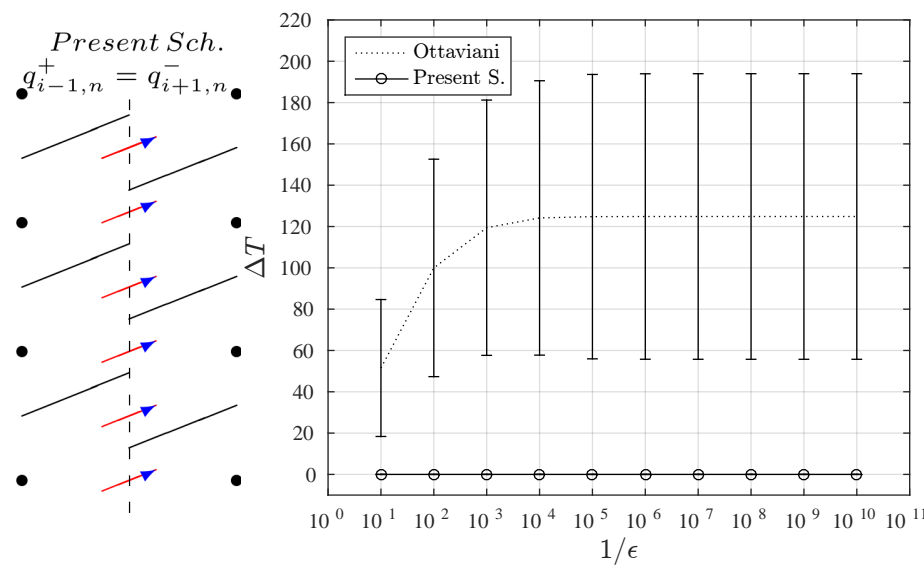

(b)

Figure 6: a) Sketches of the flux discretizations between adjacent control volumes for the Ottaviani's scheme (aligned to the grid points [9]) and the present scheme (centered to the contact surface between control volumes). (b) Plot of the difference $\Delta T$ between the averaged values of the analytical solution $T_{a}$ and of the discretized solution of Eq. 3 over the domain for different values of the anisotropy coefficient $1 / \epsilon$ with $1 / \epsilon=K \sin \left(x_{i}\right) \sin \left(y_{j}\right) . \Delta T$ is averaged when varying the inclination angle $\alpha$ for $m_{y}=8$ and $m_{x} \in[1,8]$.

The conservativity of the present scheme is ensured by the discretization of the fluxes, calculated at the center of the common faces of two adjacent CVs. It was not the case 
with the Ottaviani's scheme where the fluxes were calculated at the center of the control volume faces for each plane $\mathcal{X}_{i}$ leading to a misalignement of these between adjacent CVs, as shown on Fig. 6 (a).

The difference, denoted $\Delta T$, between averaged values over the domain of the analytical solution $T_{a}$ and the discretized solution is plotted on Figure 6 (b) for both the Ottaviani's scheme and the present one. $\Delta T$ is also averaged for different angles of inclination $\alpha$ corresponding to $m_{y}=8$ and $m_{x} \in[1,8]$. For the Ottaviani's scheme (dashed line) this difference is non zero, and the bars indicate the extreme values obtained when varying $\alpha$. With the present scheme on the contrary, the difference is zero showing the conservativity of the discretization whatever the angle $\alpha$ and the anisotropy.

\section{Numerical discretization of the boundaries}

In bounded domains when the flow in the dominant direction (parallel direction) intercepts the boundary of the physical domain, the discretization must be adapted to keep the accuracy while remaining compatible with the discretization adopted for inner nodes. For non-aligned approaches like in Ref.[3], or for some aligned approaches like in Ref.[1], in which the stencil is based on surrounding grid points, the methods keep working near the boundaries, possibly using some ghost points. However, aligned approaches, involving oriented stencils as the present work or others proposed in Refs. $[9,10,12]$ for example, require a certain number of points possibly located far outside the physical domain (the number of ghost points needed is related to $\xi$ defined in Eqs. 20 and 21), as illustrated on Fig. 7a. The weakness of such approaches is that the accuracy of the extrapolation in the $y$-direction to estimate $T^{\text {int }}$ is low. Indeed, such point may be located far outside the domain whereas solutions exhibit short-wavelengths in this direction.

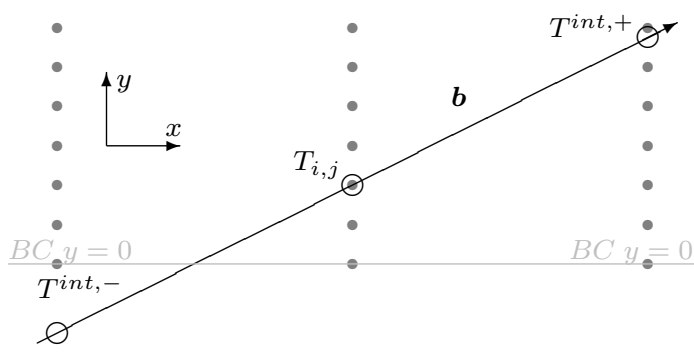

(a)

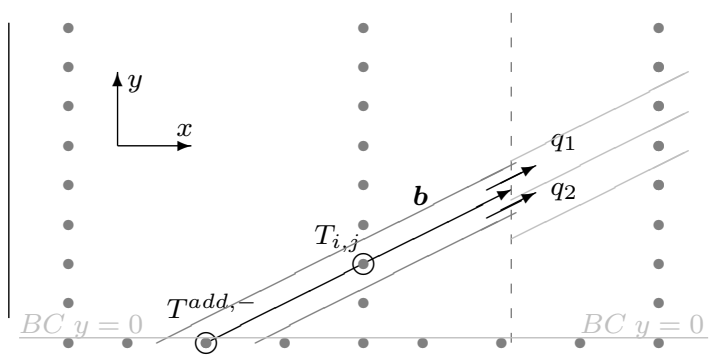

(b)

Figure 7: Numerical discretization for aligned approaches near the grid limits. (a) Classical approach with ghost points $T^{\text {int,-- }}$ located along the parallel direction and possibly far outside the domain. (b) Present method with ghost points $T^{\text {add }}$ added on the boundary of the domain.

According to what mentioned above, we propose to add ghost points directly on the boundary of the domain, as shown in Fig. 7b. Such points are needed since we may 
have at the two boundaries of the domain, depending on the resolution and the incidence value $\alpha$ :

$$
\int_{x_{i}}^{x_{i-1}} \frac{b_{y}}{b_{x}} d x<0 \quad \text { at } y=0 \quad \text { and } \quad \int_{x_{i}}^{x_{i+1}} \frac{b_{y}}{b_{x}} d x>2 \pi \quad \text { at } \quad y=2 \pi
$$

For any point $T_{i, j}$ located close to the grid limits at $y=0$ and $y=2 \pi$, extra points are added in the $x$-direction at the coordinates:

$$
x_{a d d}=i \Delta x+\int_{0}^{y_{i}} \frac{b_{x}}{b_{y}} d y \quad \text { at } \quad y=0 \quad \text { and } \quad x_{a d d}=i \Delta x+\int_{y_{i}}^{2 \pi} \frac{b_{x}}{b_{y}} d y \quad \text { at } \quad y=2 \pi
$$

These points being now located on the boundary, the value of the field may be directly obtained from the boundary conditions of the problem Eq. 3. For Dirichlet boundary condition $(\beta=0$ and $\gamma=1)$ the results is immediate. For Neumann boundary condition $(\beta=1$ and $\gamma=0)$, the derivative in the parallel direction has to be evaluated using interior grid points. In this case, we get:

$$
\begin{gathered}
T_{y=0}^{a d d}=\frac{\nabla_{\|} T_{y=0}^{a d d}\left(d_{\|, 1}+d_{\|, 2}\right)-4 T_{i, j}+T_{i+1}^{i n t}}{3} \\
T_{y=2 \pi}^{a d d}=\frac{-\nabla_{\|} T_{y=2 \pi}^{a d d}\left(d_{\|, 1}-d_{\|, 2}\right)+4 T_{i, j}-T_{i+1}^{i n t}}{3}
\end{gathered}
$$

where $d_{\|, 1}$ and $d_{\|, 2}$ (Eq. 24) are the arc lengths in the parallel direction between $T^{a d d}$ and $T_{i, j}$, and between $T_{i, j}$ and $T^{i n t,+}$, respectively, $T^{i n t,+}$ being the interpolated value of the field in the plane $\mathcal{X}_{i+1}$.

Since the values of $T^{\text {add }}$ at $y=0$ and $y=2 \pi$ are located along $\boldsymbol{b}$, the CV associated to $T_{i, j}$ (See on Fig. 2) is aligned with the control volume associated to $T^{\text {add }}$. The flux discretized using finite-differences between $T_{i, j}$ and $T^{a d d}$ remains conservative. The complete operator can be calculated by considering the fluxes balance in the CV of $T_{i, j}$, i.e.:

$$
\begin{aligned}
& \nabla \cdot[K] \nabla_{\|} T_{i, j}=\frac{Q^{+}-Q_{y=0}^{a d d}}{\frac{1}{2}\left(d_{\|, 1}+d_{\|, 2}\right)} \\
& \nabla \cdot[K] \nabla_{\|} T_{i, j}=\frac{Q_{y=2 \pi}^{a d d}-Q^{-}}{\frac{1}{2}\left(d_{\|, 1}+d_{\|, 2}\right)}
\end{aligned}
$$

where $Q_{y=0}^{a d d}$ and $Q_{y=2 \pi}^{a d d}$ are the fluxes between the added grid points and $T_{i, j}$ :

$$
Q_{y=0}^{a d d}=\frac{T_{i, j}-T_{y=0}^{a d d}}{d_{\|, 1}} \quad \text { and } \quad Q_{y=2 \pi}^{a d d}=\frac{T_{y=2 \pi}^{a d d}-T_{i, j}}{d_{\|, 1}}
$$


and $Q^{ \pm}$is the total flux considering the fluxes obtained in Eq. 11, as follows:

$$
Q^{ \pm}=\frac{1}{A_{i, j}^{ \pm}} \sum_{i, j, p} q_{i, j, p} T_{i, j} a_{p} \quad \text { where } \quad A_{i, j}^{ \pm}=\sum_{p} a_{p}
$$

In Eq. 33, the matrix product $q_{i, j, p} T_{i, j}$ gives the fluxes trough the $\mathrm{CV}$, and \pm represents the relative position into the $\mathrm{CV}$ associated to $T_{i, j}$. Note than in Eq. $34 A_{i, j}^{ \pm}$and $a_{p}$ reduce to lengths since the problem is $2-\mathrm{D}$.

\section{Grid limitations study using a manufactured solution}

The problem being highly anisotropic it is worthwhile to investigate the representation of the solution with respect to the stencil in a Cartesian grid. The results presented in this section solves the Eq. 3 with $\mu=1$ with bi-periodic boundary conditions and $1 / \epsilon=1$, considering only $K_{b}$ contribution in Eqs. 4,6 .

\subsection{Nyquist-Shannon theorem}

The Nyquist-Shannon theorem [13, 14] demonstrates that the minimal resolution $N$ in an arbitrary direction is equal to $N=2 m$, where $m$ is the highest wavenumber of the solution in this direction. Thus, this theorem provides a criterium to identify certain numerical behaviours of the solution found depending on the grid used. To illustrate this result, we have firstly performed the polynomial reconstruction of the function $f(x)=\sin \left(m_{x} x\right)$, with $m_{x}=50$. As expected from the theorem, the error made in the signal reconstruction when increasing the resolution starts to decrease exactly for $N=2 m_{x}$, and this feature is independent of the degree of interpolation. This provides the minimal resolution needed for a given wavelength.

Problem Eq. 3 with $\mu=1$ is now considered with periodic boundary conditions and for the two-dimensional manufactured solution:

$$
T_{a}(x, y)=1+\cos \left(m_{y} y+m_{x} x\right),
$$

with $m_{y}=13$ and $m_{x}=2$. This leads to an inclination angle $\alpha=8.75^{\circ}, \alpha=$ $\tan ^{-1}\left(m_{x} / m_{y}\right)$. As shown on Fig. 8, the field remains constant in the parallel direction defined by $b=(\cos \alpha, \sin \alpha, 0)$, while rapid variations can be observed in the perpendicular direction.

Tests are performed keeping the resolution fixed in the $x$-direction. The $\left\|T-T_{a}\right\|_{\mathcal{L}_{2}}$ error is plotted on Fig.9 for various numerical schemes with aligned and non-aligned interpolations, when increasing the resolution in $y$-direction:

- The asymmetric approach (not oriented stencil) [1], referred here as the classic scheme. 


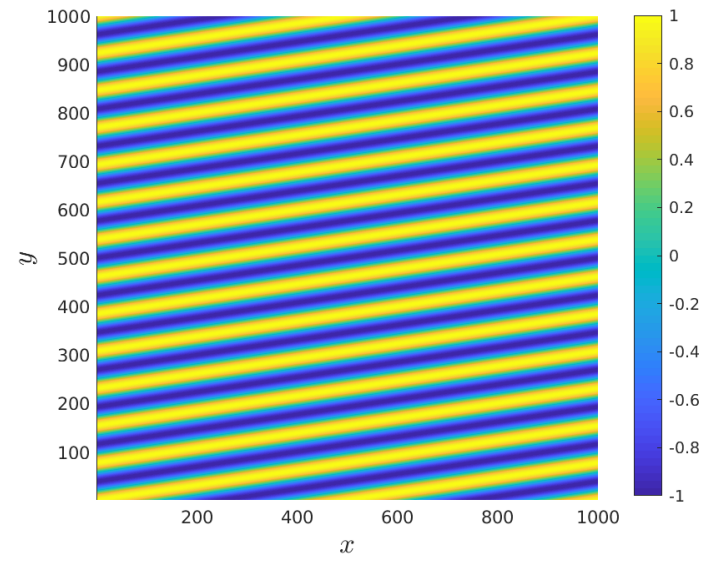

(a)

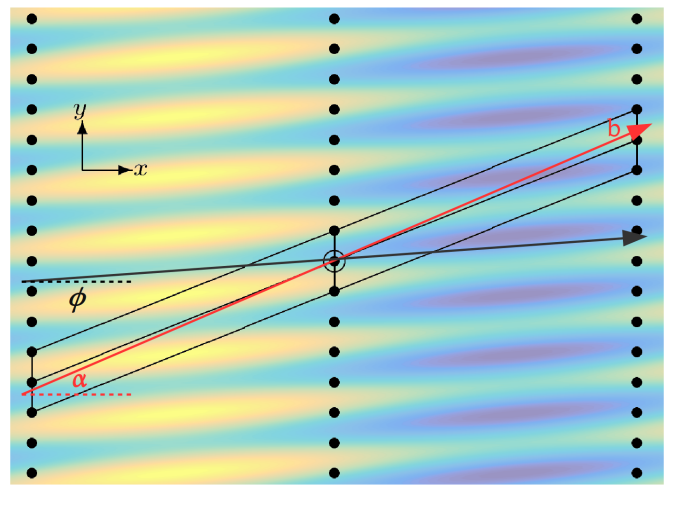

(b)

Figure 8: (a) 2D plot of the manufactured solution $T(x, y)$ [Eq. 35] with $N_{x}=N_{y}=1000$. The computational domain is $[0,2 \pi] \times[0,2 \pi]$. (b) Misalignment representation between the solution pattern $\phi$, and the parallel diffusion direction $\alpha$ out of the range Eq. 37 .

- The symmetric approach presented in Gunter et al. [3] (not oriented stencil), referred here as the Gunter's scheme.

- The aligned approach (oriented stencil) based on a second order polynomial interpolation, and a second order finite-difference scheme presented in [10, 9], referred here as the Ottaviani's scheme.

- The aligned approach (oriented stencil) based on a linear interpolation proposed in Stegmeir et al. [12], referred here as the Stegmeir's scheme.

- The present scheme with linear interpolation and oriented stencil.

\subsection{Numerical schemes with non-aligned interpolation}

Problem Eq.3 is discretized in the two $x$ and $y$ directions using both a classical finite-difference method (also known as asymmetric scheme in $[3,1]$ ) and the symmetric Gunther's scheme [3]. Stencils of both schemes are not oriented.

Fig. 9a shows the $\mathcal{L}_{2}$-norm when increasing the resolution in the $y$-direction only $\left(\mathrm{N}^{*} \mathrm{x}=32\right)$. The dotted line shows the minimal resolution expected from the NyquistShannon theorem. This allows us to relate oscillations to aliasing effects with two extrema at $N_{x} \times N_{y}=2.88 \times 10^{2}$. For grid resolutions between the dotted and dashed lines, the convergence is dominated by the discretization error in the $y$-direction. The dashed line shows the lowest error, and corresponds to a resolution where the grid and the direction of the parallel diffusion are perfectly aligned. Beyond this resolution, increasing the 
number of points in the $y$-direction does not lead to an error decrease, the error being now dominated by the discretization in the $x$-direction, which is fixed.

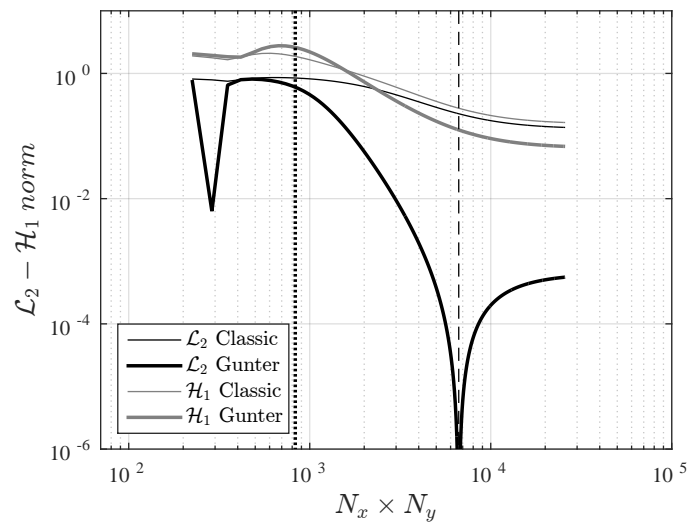

(a)

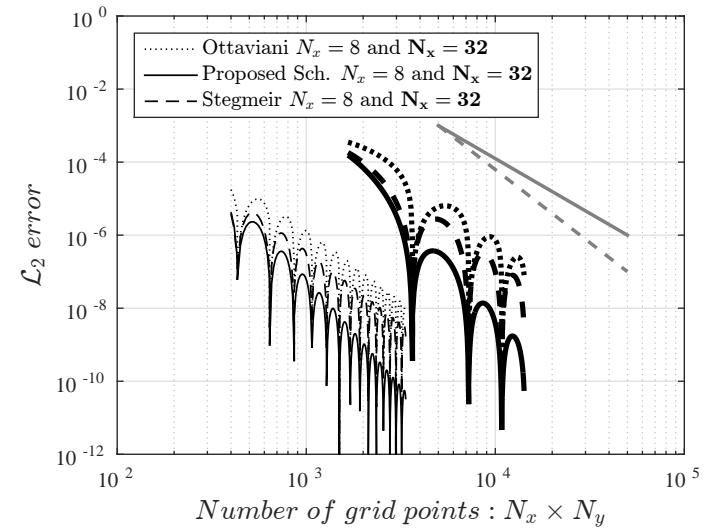

(b)

Figure 9: $\left\|T-T_{a}\right\|_{\mathcal{L}_{2}}^{2}$ and $\left\|T-T_{a}\right\|_{\mathcal{H}_{1}}^{2}$-norms when increasing resolution. (a) Non-aligned approaches: classic scheme and symmetric Gunter's scheme [3]. The resolution in the $x$ direction is fixed, with $N_{x}=32$. The dotted line corresponds to the minimal resolution prescribed by the Nyquist-Shannon theorem. The dashed line corresponds to the resolution where the grid is perfectly aligned with the direction of the parallel diffusion. (b) Aligned interpolation schemes for $N_{x}=8$ (thin lines) and $N_{x}=32$ (thick lines) for $N_{y}=[50,250]$.

To better take into account these discretization features and avoid false optimal performance points, we will consider the $\mathcal{H}_{1}$-norm, which is a upper bound of $\mathcal{L}_{2}$-norm integrating the norm of the gradient in the $x, y$-directions. Thus,

$$
\left\|T-T_{a}\right\|_{\mathcal{H}^{1}}^{2}=\left\|T-T_{a}\right\|_{\mathcal{L}^{2}}^{2}+\|\nabla T\|_{\mathcal{L}^{2}}^{2} \geqslant\left\|T-T_{a}\right\|_{\mathcal{L}^{2}}^{2}+\left\|\nabla_{x} T\right\|_{\mathcal{L}^{2}}^{2}+\left\|\nabla_{y} T\right\|_{\mathcal{L}^{2}}^{2}
$$

\subsection{Numerical schemes with aligned approach}

Tests are carried out with the present scheme together with the Ottaviani's discretization $[9,10]$ based on a 2nd-order polynomial interpolation and the Stegmeir's discretization [12] based on a linear interpolation.

Accurate interpolation in the y-direction in the calculation of the parallel derivative needs a number of grid points at least equal to the value provided by the Nyquist-Shannon theorem.

In oriented stencils to the parallel diffusion direction, the Nyquist-Shannon theorem provides the misalignment limit between the orientation angle of the local solution pattern Fig. 8, denoted by $\phi$, and the parallel diffusion direction $\alpha$, Eq.37. The NyquistShannon criterium must be verified for the values of the solution on the grid along the 
parallel direction, to accurately estimate the parallel derivative. This saves grid points in the $\mathrm{x}$-direction since the wavenumber of the solution is smaller in the parallel direction than in the $\mathrm{x}$-direction. They are trivially equal when $\alpha=0$.

$$
\tan ^{-1}\left(\frac{4 \Delta x \tan \phi-\frac{\lambda_{y, \min }}{2}}{4 \Delta x}\right) \leq \alpha \leq \tan ^{-1}\left(\frac{4 \Delta x \tan \phi+\frac{\lambda_{y, \min }}{2}}{4 \Delta x}\right)
$$

Note that the misalignment angle limit is not symmetric with respect to the diffusion direction given by $\alpha$.

The interpolation step when using oriented stencils introduces an additional error to the finite-difference scheme. Figure $9 \mathrm{~b}$ shows the oscillations due to this. Here, the number of points only varies in the $y$-direction, and the resolution in the $x$-direction is kept fixed at $N_{x}=8$ or $N_{x}=32$. The various local minima of the errors correspond to resolutions where the grids are aligned along the parallel diffusion direction, while the local maxima of the errors correspond to the largest grid-misalignment.

Note since interpolation error is proportional to $d_{\|}^{-2}=\left(\Delta x \cos ^{-1} \alpha\right)^{-2}$ using a finitedifferences discretization, Figure $9 \mathrm{~b}$ shows how a higher $x$-resolution (i.e.a lower $d_{\|}$value) increases the error of interpolation for the same number of grid points in the $y$-direction.

\section{Numerical tests in periodic domain}

Eq. 3 is solved considering only the parallel contribution in the diffusion tensors Eq. 2 .

The analytical soltuion $T_{a}$ Eq. 38 is characteristic of a realistic highly anisotropic diffusion flow as those occuring in magnetized fusion models [9]. It exhibits long structures oriented to the main diffusion direction in combination to rapid variations in the perpendicular direction (Figure 10):

$$
T_{a}(x, y)=C_{1}+C_{2} \cos \left(m_{y} y+m_{x, 1} x\right)+C_{3} \sin \left(m_{x, 2} x\right)
$$

where $C_{1}, C_{2}, m_{y}, m_{x, 1}$ and $m_{x, 2}$ are constants of the problem.

The pitch angle $\alpha$ is rewritten as:

$$
\alpha=\tan ^{-1}\left(\frac{1}{q}\right)=\tan ^{-1}\left(\frac{m_{x, 1}\left(x_{\max }-x_{\min }\right)}{m_{y}\left(y_{\max }-y_{\min }\right)}\right)
$$

Test cases have been launched for a wide range of grid points, setting the number of unknowns in each direction as multiples of two, with a maximum equal to 262144. Plotted results correspond to the lowest value of the $\mathcal{H}^{1}$-norm when varying independently the resolution in each direction, only the total number of points being fixed.

The sensitivity in the resolution of problem Eq. 3 to $\mu$ is shown on Figure 11 for various values of the parameter smaller or equal than 1 . The results show that the resolution of Eq. 3 is little sensitive to $\mu$, except for very small values where the problem becomes 


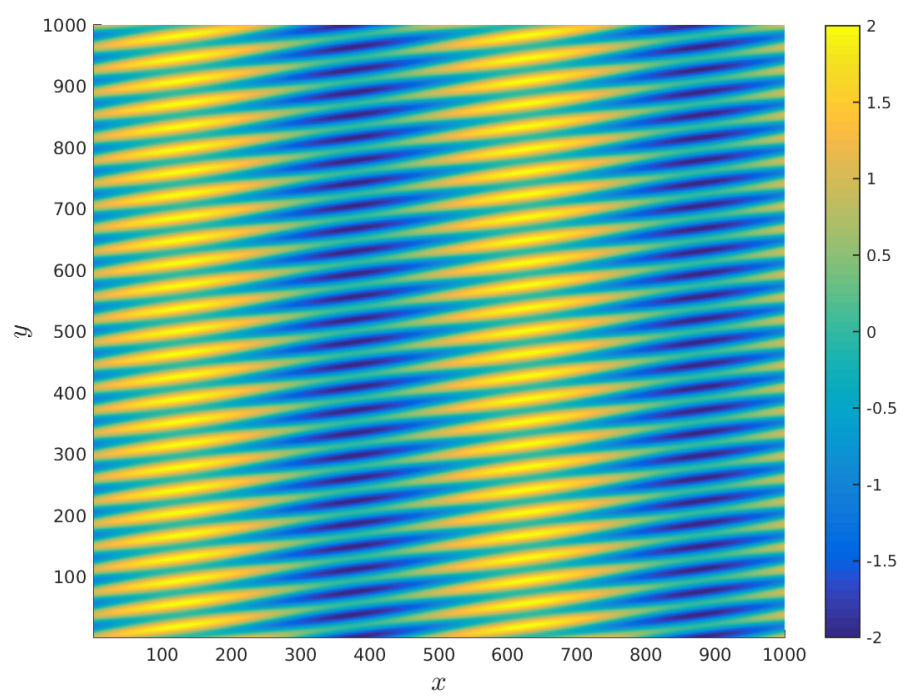

Figure 10: Plot of $T_{a}$ Eq. 38 with $C_{1}=0, C_{2}=1, C_{3}=1, m_{y}=27, m_{x, 1}=4$ and $m_{x, 2}=2$.

singular (Poisson's equation with periodic boundary conditions). In the following, we will only consider $\mu=1$ in all tests.

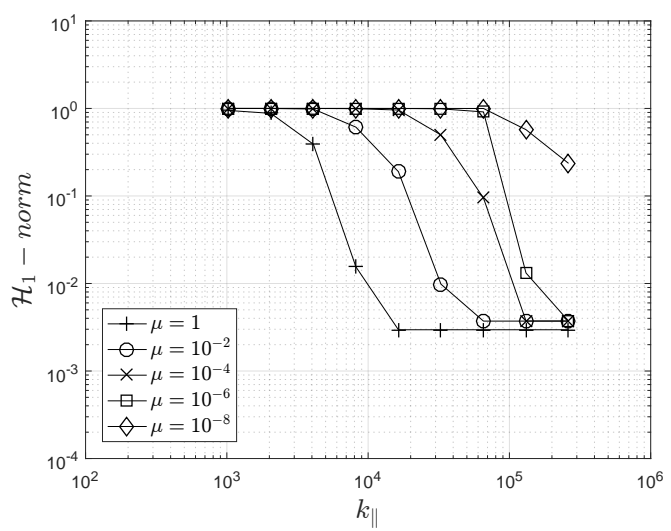

Figure 11: $H_{1}$-norm convergence in a periodic domain of the discretized solution to $T_{a}$ when increasing the resolution, for $\mu$ values smaller or equal than one, $\mu \in\left[10^{-6}, 1\right] . \alpha=\tan ^{-1}(4 / 27)$, $m_{y}=27, m_{x, 1}=4, m_{x, 2}=2$, and $1 / \epsilon=1$.

Convergence results are presented on Figure 12 for an isotropic $(1 / \epsilon=1)$ and anisotropic $\left(1 / \epsilon=10^{6}\right)$ diffusion tensor. For all cases, the plots show a better accuracy of the aligned schemes for a given number of grid points. As expected, when the 
anisotropy becomes stronger only the aligned schemes converge, Figure 12(b) and Figure 13. For a prescribed accuracy, the present scheme as well as the Stegmeir's scheme save points with respect to the Ottaviani's scheme.

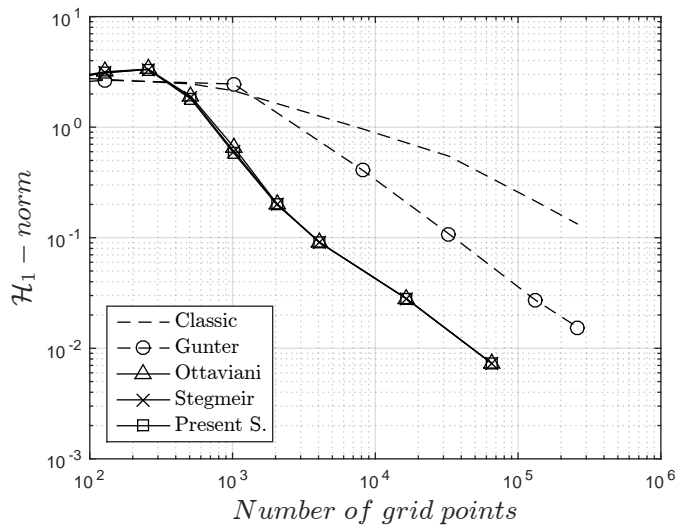

(a)

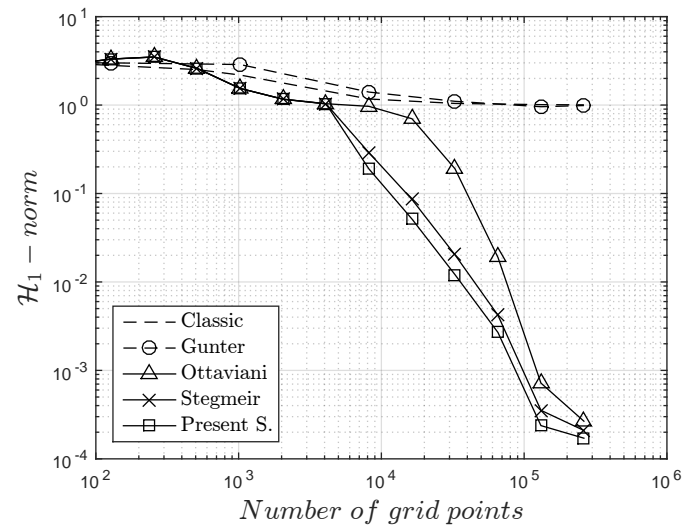

(b)

Figure 12: $H_{1}$-norm convergence in a periodic domain of the discretized solution to $T_{a}$ when increasing the resolution. (a) $1 / \epsilon=1$ and (b) $\frac{1}{\epsilon}=10^{6}$.

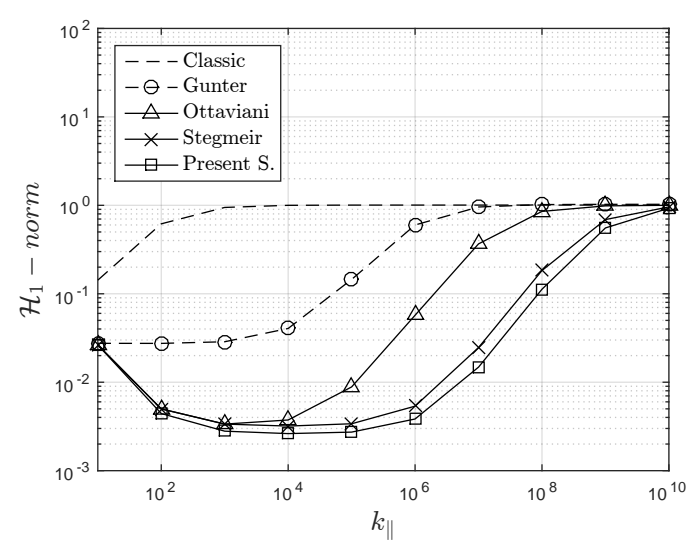

(a)

Figure 13: $H_{1}-$ norm evolution in a periodic domain with respect to the anisotropy intensity $1 / \epsilon$ for the grids leading to the lowest errors $(128 \times 4096$ for the classic scheme, $128 \times 1024$ for Gunter's scheme, and $16 \times 4096$ for all aligned approaches.

\section{Numerical tests in bounded domains}

In bounded domains, oriented stencil approaches are combined with the proposed approach near the boundary. For not oriented stencil approaches, the same approach 
is used in the whole domain. In particular, for Neumann boundary conditions, Gunter approach parallel derivative is obtained by interpolation in the grid nodes, and by crossed differentiation to the grid point.

\subsection{Elliptic problem with Dirichlet boundary condition}

The addition of aligned points in the boundary limits plays a key role in the convergence of oriented stencil approaches in highly anisotropic grids. As seen in Fig. 12, oriented stencil approaches reach the best performance when $N_{x} \ll N_{y}$, which determines a higher shift value $\xi$ for a given pitch angle $\alpha>0$. In Fig. 14a, different approaches near the boundary limits has been tested for the test case Eq. 35 in combination with presented approach for the interior grid points. Extrapolated grid points in y-direction presents a poor performance, since the rapid frequencies in this direction limits the extrapolation quality as explained in Sec. 5. The use of a not oriented approach like Gunter approach demands the use of only one ghost point in y-direction, but the $N_{x} / N_{y}$ rate is out of the limit of Nyquist-Shanon theorem for not oriented stencil approaches when oriented stencils reach the higher performance. Added points increase lightly the number of global unknowns (added points $=2 N_{x} \xi \ll N_{x} \times N_{y}$ ), and maintains the convergence founded on bi-periodic cases. Fig. 14b shows the condition number associated Fig. 14a points: the use of Gunter or extrapolated points arise the condition number in general (since these approaches requires a bigger number of unknowns in x-direction).

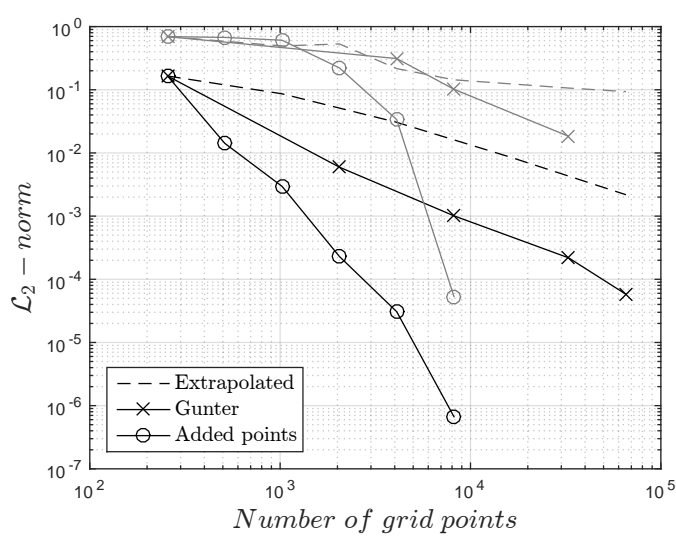

(a)

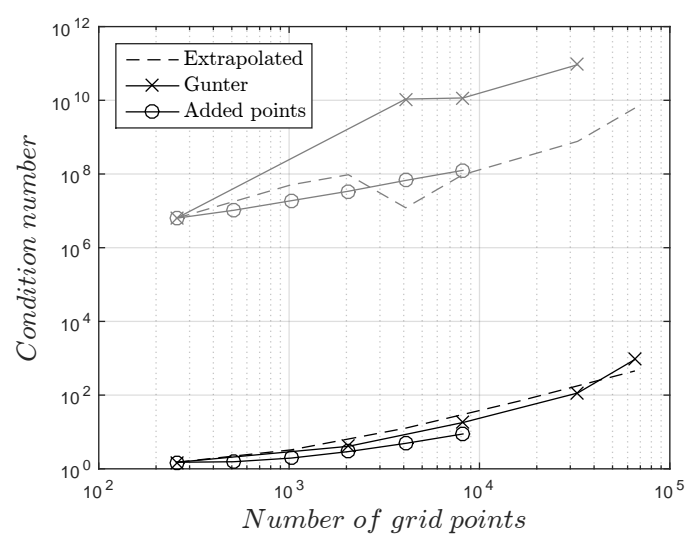

(b)

Figure 14: (a): $\mathcal{L}_{2}$-norm for $\alpha=\tan ^{-1}\left(\frac{4}{27}\right)\left(m_{y}=27 ; m_{x}=4\right)$ in Eq. 35 for Dirichlet boundary conditions with $\frac{1}{\epsilon}=10^{0}$ (black lines) and $\frac{1}{\epsilon}=10^{6}$ (gray lines) for the presented scheme in the inside grid points and combined with the specified approach near the boundary. In (b), the condition number of the numerical matrix is presented for the values of chart (a).

Precision-resolution comparison. Dirichlet boundary conditions with added aligned points on the toroidal direction in the limits. 


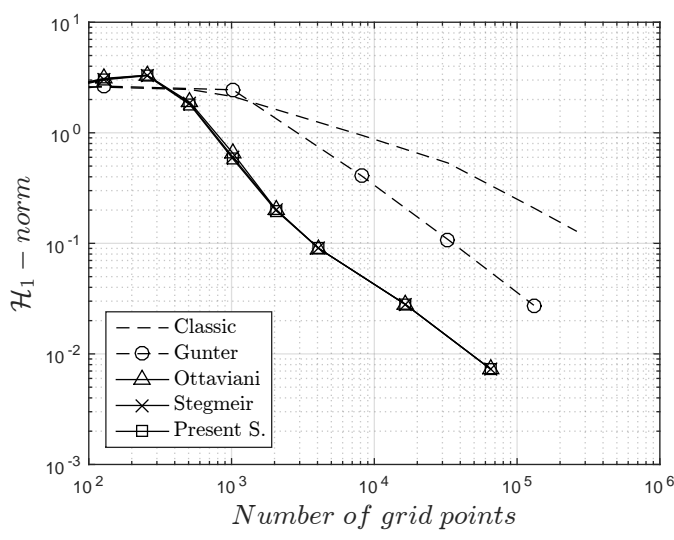

(a)

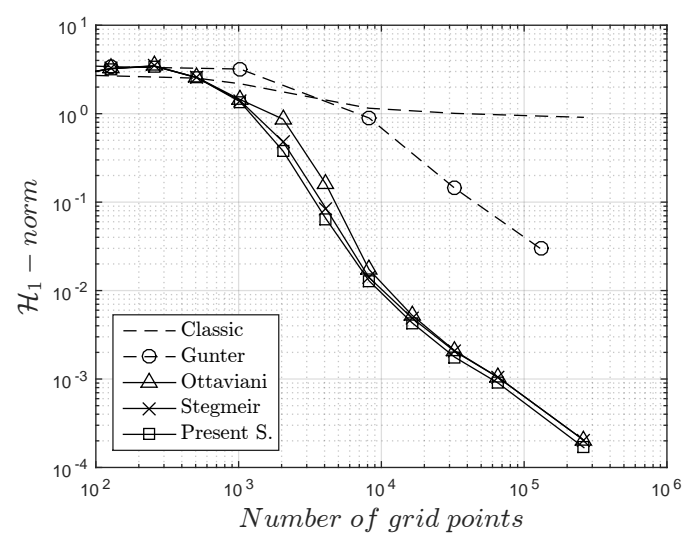

(b)

Figure 15: Minimal number of grid points for a given $H_{1}$-norm for $\alpha=\tan ^{-1}\left(\frac{4}{27}\right),\left(m_{y}=\right.$ 27; $m_{x, 1}=4$ and $m_{x, 2}=2$ ) in test case eq. 38 with Dirichlet boundary conditions: In (a) $\frac{1}{\epsilon}=10^{0}$ and (b) for $\frac{1}{\epsilon}=10^{6}$.

\subsection{Special case of the Poisson's equation with Robin boundary condition}

Poisson's equation:

$$
\begin{array}{cc}
\nabla \cdot([K] \cdot \nabla)) T=S & \text { on } \Omega \\
\frac{1}{R} \nabla_{\|} T+T=s & \text { in } \Gamma
\end{array}
$$

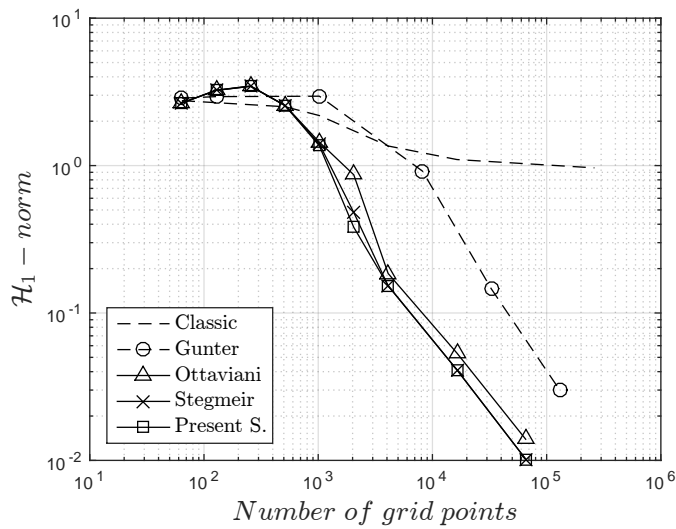

(a)

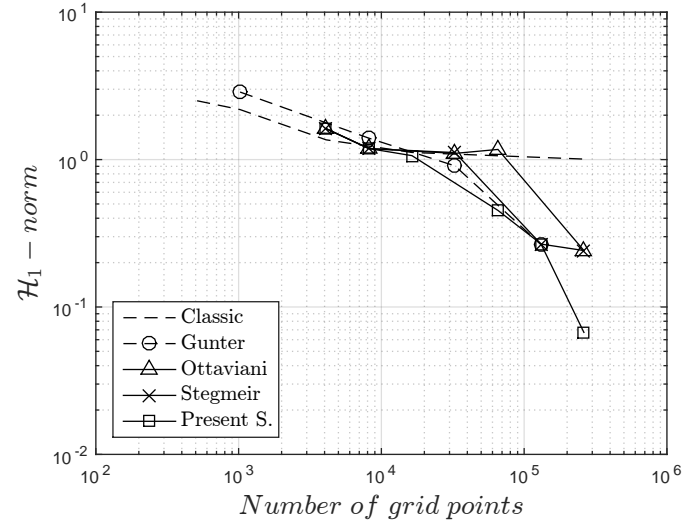

(b)

Figure 16: $\mathcal{H}_{1}$ norm for $\alpha=\tan ^{-1}\left(\frac{4}{27}\right)\left(m_{y}=27 ; m_{x, 1}=4 ; m_{x, 2}=2\right.$ in eq. 35$)$ for $R=10^{0}$ and $R=10^{-3}$ with $\frac{1}{\epsilon}=10^{0}$ in Eq. 40 .

\section{Conclusion}

[1] B. van Es; B. Koren and H. J. de Blank. Finite-difference schemes for anisotropic diffusion. J. Computational Physics, 272:526-549, 2014. 


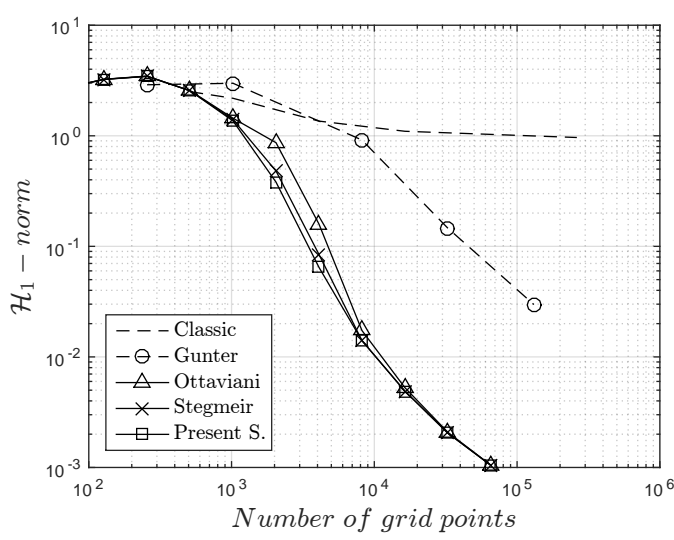

(a)

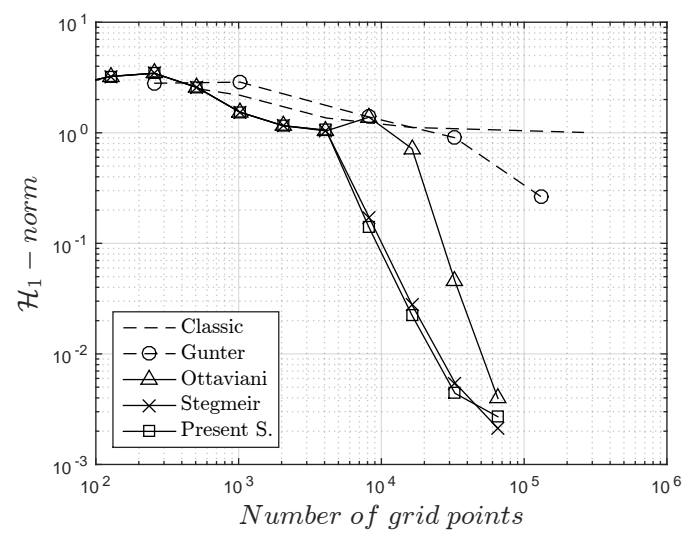

(b)

Figure 17: $\mathcal{H}_{1}$ norm for $\alpha=\tan ^{-1}\left(\frac{4}{27}\right)\left(m_{y}=27 ; m_{x, 1}=4 ; m_{x, 2}=2\right.$ in eq. 35) for $R=10^{0}$ and $R=10^{-3}$ with $\frac{1}{\epsilon}=10^{6}$ in Eq. 40 .

[2] M. Suri I. Babuska. On locking and robustness in the finite element method. J. Computational Physics, 29(5):1261-1293, 1992.

[3] S. Günter; Q. Yu; J. Krüger and K. Lackner. Modelling of heat transport in magnetised plasmas using non-aligned coordinates. J. Computational Physics, 209:354370, 2005.

[4] L. G. Margolin and M. Shashkov. Finite volume methods and the equations of finite scale: A mimetic approach. Int. J. Numer. Meth. Fluids, 56:991-1002, 2008.

[5] M. Shashkov and S. Steinberg. Support-operator finite-difference algorithms for general elliptic problems. J. Computational Physics, 118:131-151, 1994.

[6] J. M. Hyman and M. Shashkov. Approximation of boundary conditions for mimetic finite-difference methods. Computers Math. Applic., 36(5):79-99, 1998.

[7] R. M. Roberts J. E. Morel and M. Shashkov. A local support-operators diffusion discretization scheme for quadrilateral r-z meshes. J. Computational Physics, 144:17-51, 1998.

[8] P. Tamain, H. Bufferand, G. Ciraolo, C. Colin, D. Galassi, P. Ghendrih, F. Schwander, and E. Serre. The TOKAM3X code for edge turbulence fluid simulations of tokamak plasmas in versatile magnetic geometries. J. Computational Physics, 321:606-623, 2016.

[9] M. Ottaviani. An alternative approach to field-aligned coordinates for plasma turbulence simulations. Physics Letters, 375:1677 - 1685, 2011. 
[10] F. Hariri and M. Ottaviani. A flux-coordinate independent field-aligned approach to plasma turbulence simulations. Computer Physics Communications, 184:24192429, 2013.

[11] A. Arakawa. Computational design for long-term numerical integration of the equations of fluid motion: two-dimensional incompressible flow, part i. J. Computational Physics, 135:103-114, 1997.

[12] A. Stegmeir; D. Coster; O. Maj; K. Hallatschek and K. Lackner. The field line map approach for simulations of magnetically confined plasmas. Computer Physics Communications, 198:139-153, 2016.

[13] H. Nyquist. Certain topics in telegraph transmission theory. American Telephone and Telegraph Co. AIEE Winter Convention, 1928.

[14] C. E. Shannon. A mathematical theory of communication. The Bell System Technical Journal, 27:379-423, 623-656, 1948. 